\title{
Manejo del dolor: Trastorno por uso de opiáceos de prescripción médica y su relación con la práctica clínica
}

\author{
SOFÍA HERRERA A. ${ }^{1}$, AUGUSTO ROLLE P. ${ }^{2}$, MARCELA BABUL K. ${ }^{3}$, ARMANDO MALDONADO M. ${ }^{4}$, \\ MAXIMILIANO ZAMORA H. ${ }^{5}$, CLAUDIO NAZAR J. ${ }^{6}$
}

\begin{abstract}
Médico general. Interno de Medicina.

Profesor Asistente adjunto, Jefe Unidad de Adicciones, Departamento de Psiquiatría. Instructor adjunto, Programa de Medicina Paliativa y Cuidados Continuos.

Residente de Anestesiología, División de Anestesiología.

Profesor Asociado adjunto, División de Anestesiología.

Escuela de Medicina, Facultad de Medicina, Pontificia Universidad Católica de Chile.

Santiago, Chile.
\end{abstract}

Key words: Pain management, Opioid-related disorders, Analgesics, Opioids, Substance-related disorders.

\section{El manejo del dolor y su importancia}

La Asociación Internacional para el estudio del dolor (IASP) define el dolor como "una experiencia sensorial y emocional desagradable, asociada a una lesión tisular actual, potencial o descrita en términos de tal lesión"1. Esta definición intenta agrupar la percepción objetiva y subjetiva de dicho estímulo, por lo que es importante considerar como "dolor" todo aquello que el paciente dice sentir como tal, aún sin una condición física evidente ${ }^{2}$.

Según su temporalidad el dolor se clasifica en agudo o crónico, siendo este último frecuentemente descrito como aquél dolor que excede un período de 6 meses y/o aquel dolor que persiste por un período de tiempo mayor al que se requiere para que la lesión que lo originó haya sanado por completo y asimismo, cuando el dolor acompaña persistentemente a una enfermedad de curso crónico ${ }^{3,4}$.

Es de gran importancia realizar un manejo adecuado del dolor crónico debido, entre otros factores, al gran impacto que genera en la calidad de vida tanto del paciente como de su entorno más cercano, a los elevados costos asociados $\mathrm{y}$ a los efectos adversos a nivel fisiológico que produce en múltiples sistemas. Idealmente, esta tarea debe estar a cargo de un equipo de manejo del dolor, grupo multidisciplinario conformado

Los autores no refieren conflictos de interés.

Correspondencia a:

Dr. Claudio Nazar J.

Marcoleta 367, 3er piso, Hospital Clínico UC.

Tel: 26398766 - 23543270. Fax: 26327620

Código postal: 6510260. Santiago, Chile.

cenazar@med.puc.cl 
por médicos de distintas especialidades, entre los que destacan: anestesiólogos, especialistas en cuidados paliativos, psiquiatras y fisiatras en conjunto con otros profesionales de la salud: enfermeras, psicólogos, kinesiólogos y terapeutas ocupacionales cuyo objetivo es trabajar en conjunto en torno a un plan de manejo del dolor que incluya las distintas facetas de éste ${ }^{5}$.

Dentro de los fármacos utilizados para manejo del dolor y según la escalera analgésica de la Organización Mundial de la Salud (OMS), encontramos: Paracetamol, Aspirina, antiinflamatorios no esteroidales y opiáceos; estos últimos cobran gran relevancia en el tratamiento del dolor crónico; detallaremos sus principales características a continuación ${ }^{6,7}$.

\section{Opiáceos}

Los opiáceos son drogas derivadas del opio, siendo su exponente más conocido la morfina, y todos sus derivados naturales, sintéticos o semisintéticos. Actúan a nivel de receptores opioides, cuyo ligando fisiológico son las familias de péptidos encefalinas, endorfinas y dinorfinas, derivados desde distintos precursores.

A los opiáceos se les conoce, tradicionalmente, por su efecto analgésico, pero presentan una amplia variedad de efectos clínicos asociados, a nivel de sistema nervioso central, cardiovascular, respiratorio, entre otros.

Entre los receptores opioides, por su parte, existen diversos subtipos, destacando los receptores $\mathrm{Mu}(\mu)$, Kappa $(\kappa)$ y Delta $(\delta)$, con efectos diversos en relación a la modulación de acciones en los distintos sistemas fisiológicos ${ }^{8}$.

Los distintos opiáceos tienen diversa afinidad por uno o más receptores, pudiendo actuar de manera agonista, antagonista, o de acción mixta, como agonista parcial o como agonista-antagonista si actúan en más de un receptor. Eso, sumado a otras características farmacocinéticas de cada opiáceo, resulta en las indicaciones médicas en las cuales es utilizado 9 .

\section{Opiáceos de prescripción médica}

Cabe definir el concepto de opiáceo de prescripción médica, como aquellas drogas indicadas o prescritas por un médico, con un objetivo terapéutico específico y siguiendo estrictamente las indicaciones entregadas, tanto en dosis como en vía de administración y en el horario indicado. Este concepto descarta a priori drogas utilizadas en el ámbito recreativo, como es la heroína, derivado semi-sintético de la morfina.

Dentro del uso de opiáceos de prescripción médica, existen diversas indicaciones por parte del equipo médico. De esta manera, habitualmente, se reservan los opiáceos de formulación endovenosa para tratamientos intrahospitalarios y manejo del dolor en relación a procedimientos quirúrgicos y por otro lado, el manejo ambulatorio, se realiza principalmente con medicamentos administrados por vía oral o transdérmica. Destacan en un tercer grupo, los pacientes que participan de programas de cuidados paliativos, donde la vía subcutánea para administración de medicamentos adquiere especial relevancia. Cabe destacar los fármacos de administración oral como transversales a estos tres grupos de pacientes, siendo muchas veces su uso, en un inicio, intrahospitalario seguido por un manejo ambulatorio con el mismo fármaco, ya sea bajo prescripción horaria o como dosis de rescate, ante exacerbaciones del dolor.

Dentro de los opiáceos mayormente utilizados en la práctica clínica se encuentran la Morfina, Metadona, Fentanyl y Tramadol, cuyas características principales se presentan en la Tabla 1.

Con respecto al manejo de dolor crónico es relevante destacar aquellos opiáceos de uso ambulatorio dentro de los cuales, en nuestro país, destaca el Tramadol, ya sea en su formulación en gotas o tabletas y asimismo, en combinación con otros analgésicos, tales como Paracetamol, con el fin de reducir las dosis administradas de ambos fármacos ${ }^{10,11}$. Existen otros opiáceos presentes en el arsenal médico para el manejo del dolor ambulatorio, como la Codeína, opiáceo débil cuya formulación más ampliamente disponible es en jarabe, y también en tabletas que se pueden utilizar como parte del segundo escalón analgésico de las guías de la OMS. Asimismo, en otros países, existen otras formulaciones de administración oral, tales como la Oxicodona y el Tapentadol, éste último de reciente introducción al mercado en nuestro país, con disponibilidad actual dentro del arsenal terapéutico para manejo 
Tabla 1. Principales características de opiáceos de práctica clínica

\begin{tabular}{|ll|}
\hline Morfina & $\begin{array}{l}\text { Droga con vida media de } 2 \text { a } 4 \text { horas, con duración analgésica algo menor. Se metaboliza en el hígado y una } \\
\text { pequeña fracción se excreta inalterada por el rinón. Su principal metabolito (morfina-6-glucuronido) es más } \\
\text { activo que la morfina. La analgesia es dosis dependiente y su capacidad de generar dependencia física es } \\
\text { escasa. Con formulación endovenosa, oral, subcutánea y transdérmica }\end{array}$ \\
Metadona & $\begin{array}{l}\text { Posee vida media larga que varía entre } 13 \text { a } 100 \text { horas, con duración analgésica de } 4 \text { a } 8 \text { horas. Su elimina- } \\
\text { ción es prolongada por lo que existe riesgo de acumular metabolitos y es droga de elección cuando se desea } \\
\text { prescribir una dosis diaria. Principal uso en manejo de dolor crónico y prevención de síndrome de abstinen- } \\
\text { cia a opiáceos. Con formulación endovenosa, oral, subcutánea y transdérmica }\end{array}$ \\
Fentanyl & $\begin{array}{l}\text { Con vida media } 2 \text { a } 4 \text { horas, más liposoluble y potente, pero con menor duración en dosis analgésicas que } \\
\text { morfina. Primariamente metabolizado en el hígado. Norfentanyl, primer metabolito activo, detectable en } \\
\text { orina hasta } 48 \text { horas después. Con formulación endovenosa, intratecal y transdérmica, ésta última relevante } \\
\text { para uso en dolor crónico } \\
\text { Analgésico central de acción binaria, posee mecanismo analgésico opioide y otro no mediado por receptor } \\
\text { opioides. Con metabolito activo de su excreción hepática (70\%) y con una fracción restante (30\%) de ex- } \\
\text { creción renal inalterada. Con bajo potencial adictivo intrínseco debido a su escasa unión a receptores } \mu 1 \text { y } \\
\text { débil a receptores } \delta \text { y } \kappa \text {. Con formulación endovenosa y vía oral (gotas y comprimidos) y oral de liberación } \\
\text { sostenida }\end{array}$ \\
\hline
\end{tabular}

Nota. Adaptado de Guerrero et. al. Dolor. Aspectos básicos y clínicos ${ }^{4}$ y Opioid analgesics. Miller's Anhestesia ${ }^{14}$

del dolor ${ }^{12}$. Entre otros opiáceos de administración oral encontramos la Naltrexona y Naloxona, antagonistas de receptores opioides tipo $\mathrm{Mu}$, los que son principalmente utilizados en contexto de adicción a opiáceos y para revertir efectos adversos de opiáceos ${ }^{13}$. En el ámbito ambulatorio, la vía transdérmica es utilizada como tratamiento de segunda línea para dolor crónico de difícil manejo, destacando los parches de Buprenorfina y de Fentanyl. En la misma línea de dolor crónico de difícil manejo, están los opiáceos potentes, de formulación endovenosa o subcutánea, utilizados principalmente cuando la etiología del dolor crónico es oncológico. Destacan en este punto la Morfina, Fentanyl y Metadona, anteriormente mencionados.

Es importante considerar que los opiáceos, como todo fármaco, no están exentos de efectos adversos los cuales pueden ser derivados de su utilización en forma aguda como son: sedación, prurito, depresión respiratoria, náuseas y vómitos, constipación y retención urinaria, entre otros o crónica donde, cabe destacar, el trastorno por uso de opiáceos como uno de los efectos adversos más temidos y que, actualmente, se encuentra en aumento, provocando múltiples consecuencias tanto a nivel individual como poblacional.

\section{Trastorno por uso de opiáceos de prescripción médica}

En la literatura, es posible encontrar diversas definiciones del trastorno por uso de opiáceos de prescripción médica. Principalmente, se refiere al uso sin prescripción médica de estas sustancias como también a su uso con prescripción médica, pero en un patrón distinto al indicado por el médico para el tratamiento de una patología específica, con el fin de alcanzar la experiencia o efectos asociados que estos provocan ${ }^{15-17}$.

Los criterios DSM-V definen el trastorno por uso de sustancias, esencialmente, como un conjunto de síntomas cognitivos, conductuales y psicológicos en un individuo que continúa utilizando una sustancia a pesar de problemas relacionados con su consumo. Siendo necesario un período de doce meses y dos de los criterios ahí presentes, como mínimo, para realizar el diagnóstico; además, divide su severidad según el número de síntomas, en leve, moderado y severo. En el caso específico de los opiáceos estos criterios incluyen su consumo en cantidades mayores o durante un período más largo al que inicialmente se pretendía, gasto de mayor tiempo en obtener, consumir y recuperarse de sus efectos, "craving", fuerte deseo o urgencia de consumir 
opiáceos, uso recurrente de estos en situaciones en que es físicamente peligroso, su uso continuo a pesar de notar problemas físicos, psicológicos $\mathrm{y} /$ sociales, la presencia de tolerancia y abstinencia, entre otros ${ }^{18}$.

Con respecto a la magnitud de este trastorno, se ha convertido en un problema significativo a nivel mundial ${ }^{19,20}$. La encuesta de salud y abuso de drogas de los Estados Unidos del año 2013, señala que, 24,6 millones $(9,4 \%)$ de norteamericanos mayores de 12 años son usuarios de drogas ilícitas y de estos, 4,5 millones $(2,5 \%$ de la población) abusa de fármacos para el manejo del dolor, dentro de los cuáles se incluyen los opiá$\operatorname{ceos}^{21}$.

En Chile, el más reciente estudio nacional de drogas en población general publicado el año 2013, no incluía cifras con respecto al trastorno por uso de opiáceos. El último estudio nacional en incluir el abuso de opiáceos data del año 2006. En éste, el consumo no sobrepasaba el $0,1 \%$ de la población e incluyendo todos los analgésicos sin prescripción médica, llegaba a un $0,4 \%$ de la población $^{22,23}$.

Asimismo, esta falta de evidencia con respecto al trastorno por uso de opiáceos, está presente a nivel internacional; una revisión sistemática publicada recientemente acerca del uso de opiáceos de prescripción médica para el lumbago crónico determinó que no existían estudios clínicos randomizados que evaluaran el trastorno por uso de opiáceos en estos pacientes; sin embargo, se encontraron estudios observacionales que advertían un riesgo aumentado en estos pacientes. Las cifras de abuso y dependencia iban desde un $0,7 \%$ en pacientes en terapias con dosis bajas hasta un $6,1 \%$ en terapias con dosis altas ${ }^{24}$. Cifras similares se evidencian en la literatura variando según los criterios utilizados para diagnosticar el trastorno por uso de opiáceos y el escenario de atención: primaria versus hospitalaria ${ }^{16,25}$. Es así como otra revisión sistemática en pacientes con dolor crónico señala que entre un 21 a $29 \%$ de los pacientes presentaría un uso diferente al prescrito por el médico y las tasas de dependencia variarían entre un 8 a $12 \%{ }^{26}$.

Cabe destacar que éste no es sólo un problema que afecta a pacientes con dolor crónico, sino que también son utilizados en forma recreativa por jóvenes. En este grupo etario, constituyen los fármacos con mayor uso sin prescripción médi- ca. Asimismo, datos de la encuesta nacional de salud y uso de drogas de los Estados Unidos de América revelan que hasta un $26 \%$ de los jóvenes entre 18 y 25 años han dado un mal uso a opiáceos de prescripción médica a lo largo de su vida $^{27}$.

\section{Relación con la práctica clínica}

El uso de opiáceos en la práctica clínica ha aumentado en forma dramática durante las últimas décadas. Sólo en el año 2012 se estima que se hicieron 62 millones de prescripciones de opiáceos en los Estados Unidos. Esto debido, en parte, a la liberación de su uso para tratamiento del dolor crónico no oncológico por parte de la legislación, la percepción de que el dolor crónico fue por años subdiagnosticado y subtratado, la inclusión de estos fármacos en las nuevas guías clínicas para manejo del dolor y el marketing de la industria farmacéutica ${ }^{17,20,28}$.

En torno a su uso para tratamiento del dolor crónico, éste se basa en la Escalera Analgésica de la OMS, publicada en 1986 y que luego ha sido sometida a algunas modificaciones para grupos específicos de pacientes y tipos de dolor. Esta considera la intensidad del dolor, percibida por el paciente, con el fin de indicar la prescripción analgésica adecuada en forma escalonada, dividiendo así en tres escalones el manejo analgésico. El Tramadol, principal opiáceo de uso oral en Chile para manejo del dolor, se utiliza desde el segundo escalón, en paralelo a analgésicos no opioides y a coadyuvantes específicos según el tipo de dolor a tratar ${ }^{6}$. Es así como, por ejemplo, la guía de práctica clínica del Plan de Garantías Explícitas en Salud (GES) para tratamiento médico de la artrosis de cadera y/o rodilla, incluye opiáceos tales como Tramadol o Codeína, en caso de no responder a la primera línea de tratamiento farmacológico y ambiental ${ }^{29}$. De esta manera, opiáceos débiles pueden ser utilizados de manera adicional a Paracetamol o antiinflamatorios no esteroidales, ante la falta de respuesta a éstos o contraindicaciones en su uso. Es así como los opiáceos tienen un espacio definido en la terapia del dolor de tipo crónico, sea éste oncológico o no, con la posibilidad de ser prescritos por cualquier médico.

Además del amplio uso que se le otorga a los 
opiáceos de prescripción médica, otro aspecto relevante es la relativa facilidad para iniciar y mantener su uso, debido a su formulación vía oral, en el caso de Tramadol y Codeína y en ocasiones, debido a la falta de rigurosidad con respecto a la receta médica retenida necesaria para adquirir estos fármacos. Esto facilita, por un lado, su prescripción médica en el ámbito ambulatorio y por otro lado, el consumo mantenido por los pacientes, sea éste bajo indicación médica o no. Un estudio realizado con datos provenientes de la encuesta de salud y abuso de drogas de los Estados Unidos revela que a mayor regularidad en el consumo de opiáceos, la fuente más frecuente de obtención de éstos es por medio de prescripción médica al mismo paciente y que en grupos de consumo menos regular, predomina la obtención de estos fármacos por medio de amigos o familiares que a su vez los obtienen por prescripción médica ${ }^{30}$.

Por lo anterior, la presencia de trastorno por uso de opiáceos debe ser evaluado de manera activa, en todo paciente con uso de opiáceos y en mayor medida en pacientes con factores de riesgo; dentro de los cuales destacan: la presencia de abuso de sustancias previo, la presencia de patologías psiquiátricas y de características demográficas como son: falta de oportunidades económicas, cambio en la normativa con respecto a estos fármacos y a su disponibilidad ${ }^{31-33}$.

\section{Consecuencias del aumento de prescripciones de opiáceos}

El aumento en la disponibilidad de opiáceos de prescripción médica ha llevado a un incremento en el trastorno por uso de opiáceos durante las últimas décadas. Se ha evidenciado un aumento de pacientes que consultan por notar dependencia a estas sustancias y un aumento de ingresos para tratamiento debido a esta causa, los cuales se estima se han quintuplicado durante las últimas dos décadas ${ }^{28,31}$.

Asimismo, se ha evidenciado un aumento en emergencias médicas por uso de opiáceos; según cifras de la Red de Alerta de Abuso de drogas de los Estados Unidos éstas han aumentado en un $183 \%$ entre los años 2004 y 2011 . Siendo responsables del $29,4 \%$ de las emergencias médicas relacionadas con consumo de drogas ${ }^{17,28}$.
Otra consecuencia de gran relevancia es el aumento de muertes relacionadas al uso de opiáceos de prescripción médica a nivel mundial $^{19,34}$. Se estima que sólo durante el año 2010 hubo 16.651 muertes relacionadas con el uso de opiáceos en los Estados Unidos. Las causas de muerte descritas son, principalmente, sobredosis y accidentes asociados, dentro de estos, destacan los accidentes automovilísticos ${ }^{20,28,35}$.

Asimismo, se ha descrito un aumento en la tasa de suicidios mediante el uso de opiáceos de prescripción médica ${ }^{20,28}$. Según cifras de la Red de alerta de abuso de drogas de los Estados Unidos, se estima que hubo 200.000 visitas a servicios de urgencia por intentos suicidas relacionados al consumo de drogas y de éstas, la gran mayoría, correspondía a drogas de prescripción médica y refieren que estas prácticas han aumentado en un 41\% entre los años 2004 y $2011^{17}$.

Es así como se estima que, hoy en día, la muerte por sobredosis de opiáceos de prescripción médica, excede a las muertes por heroína y cocaína combinadas ${ }^{36}$.

\section{Conclusiones}

El manejo del dolor crónico es transversal a las distintas especialidades médicas y de vital relevancia debido a los costos que genera en salud y al impacto en la calidad de vida de los pacientes. Los opiáceos son un pilar importante de esta tarea, sin embargo, presentan efectos adversos dentro de los cuales destaca el trastorno por uso de opiáceos, el cual constituye un problema de salud pública a nivel mundial.

Como médicos debemos estar conscientes de que este aumento en la prevalencia se ha visto relacionado estrechamente al aumento de prescripciones de estos fármacos.

Por lo mismo, es de suma relevancia asesorar la presencia de un uso diferente al indicado o que constituya criterios para un trastorno por uso de opiáceos en todos los pacientes que utilizan estos fármacos y sobre todo en aquellos que presentan factores de riesgo; asimismo, considerar estos últimos en la decisión de iniciar un tratamiento con opiáceos.

Consideramos necesario capacitar en torno al tema a aquellos médicos que indiquen frecuentemente estos fármacos en su práctica clínica $\mathrm{y}$, de 
pesquisar un patrón de abuso/dependencia, derivar a especialistas en adicciones para tratamiento.

Finalmente, es necesario contar con estudios de prevalencia de trastorno por uso de opiáceos $\mathrm{y}$ de las consecuencias que esto provoca en nuestro país, ya que puede constituir un problema que esté siendo escasamente diagnosticado y tratado.

\section{Referencias}

1. Merskey H. Psychological aspects of pain. Postgrad Med J 1968 Apr;44(510):297-306.

2. Chapman CR. Psychological aspects of pain patient treatment. Arch Surg 1977 Jun;112(6):76772.

3. Bonica JJ. The management of pain of cancer. J Mich State Med Soc 1953 Mar;52(3):284-90.

4. Guerrero MG, Lacassie H. Fármacos analgésicos. Dolor. Aspectos básicos y clínicos. Ediciones Universidad Católica de Chile. Santiago de Chile; 2004. pp. 144-55.

5. Gordon DB, Dahl JL, Miaskowski C, McCarberg B, Todd KH, Paice JA, et al. American pain society recommendations for improving the quality of acute and cancer pain management: American Pain Society Quality of Care Task Force. Arch Intern Med 2005 Jul;165(14):1574-80.

6. N, K, WHO Normative Guidelines on Pain Management. Geneva: World Health Organization; 2007.

7. Puebla F. Tipos de dolor y escala terapéutica de la OMS. Dolor iatrogénico. Oncologia 2005;28(3):139-43.

8. Jamison RN, Mao J. Opioid Analgesics. Mayo Clin Proc 2015 Jul;90(7):957-68.

9. Ballantyne JC. Opioid therapy in chronic pain. Phys Med Rehabil Clin N Am 2015 May;26(2):20118.

10. Grond S, Sablotzki A. Clinical pharmacology of tramadol. Clin Pharmacokinet
2004;43(13):879-923.

11. Nossaman VE, Ramadhyani U, Kadowitz PJ, Nossaman BD. Advances in perioperative pain management: use of medications with dual analgesic mechanisms, tramadol \& tapentadol. Anesthesiol Clin 2010 Dec;28(4):647-66.

12. Power I. An update on analgesics. Br J Anaesth 2011 Jul;107(1):19-24.

13. Helm S, Trescot AM, Colson J, Sehgal N, Silverman S. Opioid antagonists, partial agonists, and agonists/antagonists: the role of office-based detoxification. Pain Physician 2008 MarApr;11(2):225-35.

14. Fukuda K. Opioid analgesics. In: Miller R, editor. Miller's Anesthesia. Philadelphia: Churchill Livingstone; 2014. pp. 887-903.

15. Degenhardt L, Bucello C, Mathers B, Briegleb C, Ali H, Hickman M, et al. Mortality among regular or dependent users of heroin and other opioids: a systematic review and meta-analysis of cohort studies. Addiction 2011 Jan;106(1):3251.

16. Degenhardt L, Bruno R, Lintzeris N, Hall W, Nielsen S, Larance B, et al. Agreement between definitions of pharmaceutical opioid use disorders and dependence in people taking opioids for chronic non-cancer pain (POINT): a cohort study. Lancet Psychiatry 2015 Apr;2(4):31422.

17. Administration S.A.a. M.H.S. Drug Abuse Warning Network, 2011: National Estimates of
Drug-Related Emergency Department Visits. 201330 de mayo de 2015]; Available from: http://www.samhsa.gov/

18. Association AP. Substancerelated and addictive disorders. Diagnostic and statistical manual of mental disorders. 5 th ed. Washington (D.C.): American Psychiatric Association; 2013. pp. 541-50.

19. Degenhardt L. Benefits and Risks of Pharmaceutical Opioids: Essential Treatment and Diverted Medication - a Global Review of Availability, Extramedical Use, Injection and the Association with HIV. National Drug \& Alcohol Research Centre; 2008.

20. Manchikanti L, Helm S 2nd, Fellows B, Janata JW, Pampati V, Grider JS et al. Opioid epidemic in the United States. Pain Physician 2012 Jul;15(3 Suppl):ES9-38.

21. Results from the 2013 National Survey on Drug Use and Health: Summary of National Findings. 201430 de mayo de 2015]; Available from: http://www. samhsa.gov/

22. CONACE, Séptimo estudio nacional de drogas en población general, 2006, 2007, Ministerio del Interior y Seguridad Pública Gobierno de Chile, Santiago de Chile.

23. SENDA, Décimo estudio nacional de drogas en población general, 2012, 2013, Ministerio del Interior y Seguridad Pública Gobierno de Chile.

24. Chou R, Turner JA, Devine EB, Hansen RN, Sullivan SD, Blazi- 
na I, et al. The effectiveness and risks of long-term opioid therapy for chronic pain: a systematic review for a National Institutes of Health Pathways to Prevention Workshop. Ann Intern Med 2015 Feb;162(4):276-86.

25. Palmer RE, Carrell DS, Cronkite D, Saunders K, Gross DE, Masters E, et al. The prevalence of problem opioid use in patients receiving chronic opioid therapy: computer-assisted review of electronic health record clinical notes. Pain 2015 Jul;156(7):1208-14.

26. Vowles KE, McEntee ML, Julnes PS, Frohe T, Ney JP, van der Goes DN. Rates of opioid misuse, abuse, and addiction in chronic pain: a systematic review and data synthesis. Pain 2015 Apr;156(4):569-76.

27. Lord S, et al. Nonmedical use of prescription opioids and stimulants among student pharmacists. J Am Pharm Assoc (2003), 2009; 49(4): 519-528.

28. Dart RC, Severtson SG, BucherBartelson B. Trends in opioid analgesic abuse and mortality in the United States. N Engl J Med 2015 Apr;372(16):1573-4.

29. Guía clínica para el tratamiento médico en personas de 55 años y más con artrosis de cadera y/o rodilla leve o moderada. Serie guías clínicas MINSAL, 2009.

30. Jones CM, Paulozzi LJ, Mack KA. Sources of prescription opioid pain relievers by frequency of past-year nonmedical use United States, 2008-2011.

JAMA Intern Med 2014 May;174(5):802-3.

31. Goldner EM, Lusted A, Roerecke M, Rehm J, Fischer B. Prevalence of Axis-1 psychiatric (with focus on depression and anxiety) disorder and symptomatology among non-medical prescription opioid users in substance use treatment: systematic review and meta-analyses. Addict Behav 2014 Mar;39(3):520-31.

32. Jack H, Masterson AR, Khoshnood K. Violent conflict and opiate use in low and middleincome countries: a systematic review. Int J Drug Policy 2014
Mar;25(2):196-203.

33. Turk DC, Swanson KS, Gatchel RJ. Predicting opioid misuse by chronic pain patients: a systematic review and literature synthesis. Clin J Pain 2008 JulAug;24(6):497-508.

34. Pierce M, Bird SM, Hickman M, Millar T. National record linkage study of mortality for a large cohort of opioid users ascertained by drug treatment or criminal justice sources in England, 20052009. Drug Alcohol Depend 2015 Jan;146:17-23.

35. Degenhardt L, Randall D, Hall W, Law M, Butler T, Burns L. Mortality among clients of a state-wide opioid pharmacotherapy program over 20 years: risk factors and lives saved. Drug Alcohol Depend 2009 Nov; 105(12):9-15.

36. Centers for Disease Control and Prevention (CDC). Vital signs: overdoses of prescription opioid pain relievers-United States, 1999-2008. MMWR Morb Mortal Wkly Rep 2011 Nov;60(43):1487-92. 